\title{
Combining chloroquine with RAD001 inhibits tumor growth in a NEN mouse model
}

\author{
Shani Avniel-Polak', Gil Leibowitz', Victoria Doviner², David J Gross and Simona Grozinsky-Glasberg' \\ ${ }^{1}$ Neuroendocrine Tumor Laboratory, Endocrinology \& Metabolism Department, Hadassah-Hebrew University Medical Center, Jerusalem, Israel \\ 2Department of Pathology, Shaare Zedek Medical Center, Jerusalem, Israel
}

Correspondence should be addressed to S Grozinsky-Glasberg: simonag@hadassah.org.il

\begin{abstract}
Patients with neuroendocrine neoplasms (NENs) often require systemic treatment, which is frequently limited by the emergence of drug resistance. mTOR inhibitors (mTORi), such as RAD001 (everolimus), have been shown to inhibit neoplasm progression. mTORi stimulates autophagy, a degradation pathway that might promote the survival of neoplasm cells that are exposed to anti-cancer therapy. Chloroquine (CQ), a well-known anti-malarial and anti-rheumatic drug, suppresses autophagy. Based on our previous results, we hypothesized that CQ may enhance the anti-tumorigenic effects of mTORi by inhibiting autophagy and we aimed to examine the anti-tumorigenic effect of CQ, alone or in combination with RAD001. We established a NEN subcutaneous xenograft mouse model and evaluated the effect of the drugs on tumor growth, mTOR pathway, autophagy and apoptosis. CQ alone and in combination with RAD001 significantly decreased neoplasm volume. Histopathological analysis revealed that the combination of CQ and RAD001 markedly inhibited mTOR activity and neoplasm cell growth, along with accumulation of autophagosomes and increased apoptosis. In conclusion, CQ enhances the anti-tumorigenic effect of RAD001 in vivo by inhibiting autophagy. Clinical trials addressing the effects of CQ therapy on neoplasm progression in patients with NENs, mainly in those treated with mTORi, are warranted.
\end{abstract}

\author{
Key Words \\ - autophagy \\ - mTOR inhibitors \\ - chloroquine \\ - RAD001 \\ - neuroendocrine neoplasms
}

\section{Introduction}

Neuroendocrine neoplasms (NENs) constitute a heterogeneous and rare family of tumors that arise from cells of the diffuse endocrine system dispersed throughout the body, with an increasing annual incidence ( 6.9/100,000 people) (Dasari et al. 2017). The majority is well-differentiated and slow growing, but at least 50\% are metastatic at diagnosis (Oberg 2005, 2011). Surgical excision with curative intent is usually limited to a small group of patients with localized disease (Oberg 2005). Whereas a variety of systemic therapeutic options are available for patients with unresectable NENs (e.g., somatostatin analogues, peptide receptor radio-ligand therapy, biological agents, etc.), they eventually fail due to drug resistance, resulting in neoplasm progression (Oberg 2005, Grozinsky-Glasberg et al. 2008).

NENs have been shown to have alterations in signal transduction pathways, such as the PI3K/Akt/mTOR pathway (Altomare \& Testa 2005). mTOR (mechanistic Target Of Rapamycin) is a serine/threonine proteinkinase and a catalytic subunit of two structurally and functionally distinct complexes, mTORC1 and mTORC2. mTOR plays a central role as a regulator of cell growth and proliferation, and is constitutively activated in NENs (Grozinsky-Glasberg \& Pavel 2012). It is well known that 
treatment with mTORC1 inhibitors (such as rapamycin and its analogues) significantly suppresses the proliferation of NEN cells of different origin (Grozinsky-Glasberg et al. 2010, Avniel-Polak et al. 2015).

However, it has been shown that mTORC1 prevents the generation of autophagosomes and the initiation of autophagy through phosphorylation of ULK1; the administration of mTORi such as RAD001 inhibits mTORC1, preventing phosphorylation of ULK1, and therefore resulting in acceleration of autophagy. Autophagy is a highly conserved, genetically controlled cell-survival pathway, stimulated in response to stress (e.g., starvation, hypoxia, etc.) (Liu et al. 2017), during which cytoplasmic components (misfolded proteins or damaged organelles) are directed to lysosomes for degradation and eventual recycling (Cecconi \& Levine 2008, Ganley et al. 2009, Hosokawa et al. 2009, Jung et al. 2009, Peterson et al. 2009, Yang et al. 2011, Riahi et al. 2016). Inhibition of mTOR by rapamycin and its derivative RAD001 (everolimus) stimulates autophagosome generation and flux, thereby promoting tumorigenesis (Mazure \& Pouyssegur 2010, Yang \& Klionsky 2010, Yang et al. 2011, Bachar-Wikstrom et al. 2013b, Czarny et al. 2015, Fang et al. 2015).

Interestingly, it appears that autophagy is in a mutual relation with the process of apoptosis, and that this interconnection is context-dependent; it has been shown that autophagy may facilitate apoptosis or necrosis (Marino et al. 2014). Therefore, autophagy has been exploited as a possible therapeutic strategy (O'Reilly et al. 2006, Amaravadi et al. 2016) in different tumor models, including glioma, glioblastoma, neuroblastoma and breast cancer, and in some NEN models (Amaravadi et al. 2007, Degtyarev et al. 2008, Fan et al. 2010, Xu et al. 2011, Hong et al. 2013, Seitz et al. 2013, Weckman et al. 2015). Chloroquine (CQ), commonly used in the treatment of malaria and of rheumatic autoimmune disorders, inhibits lysosomal hydrolase activity and consequently autophagy. CQ has lysosomotropic properties, increasing the lysosomal $\mathrm{pH}$ by inhibiting H+-ATPase, thereby inhibiting autophagosome-lysosome fusion and lysosomal degradation (Rubinsztein et al. 2012).

We have previously shown in an in vitro model that treatment with CQ alone or together with mTORi exerts a robust inhibitory effect on a pancreatic NEN cell line (BON1) proliferation along with increased apoptosis (Avniel-Polak et al. 2015). In the present study, we extended our findings and studied the effect of treatment with
$\mathrm{CQ}$, alone or in combination with RAD001 (everolimus), on NEN progression in vivo using a BON1 subcutaneous xenograft mouse model.

\section{Materials and methods}

\section{BON1 cell line}

The pancreatic-carcinoid-derived cell line, BON1, was cultured in DMEM/F12 (1:1) medium (Biological Industries, Beit-Haemek, Israel). The medium was supplemented with 10\% FCS, 1\% penicillin/streptomycin and L-glutamine (Biological Industries). All cells were cultured at $37^{\circ} \mathrm{C}$ in a $5 \% \mathrm{CO} 2$ atmosphere. The cells were grown in 10-cm plates and passaged once every 3-4 days on a 1:3 split.

\section{Reagents}

The antibodies used were as follows: anti-phospho-S6 ribosomal protein (Ser240/244); anti-LC3-II and anti SQSTM1/P62 (Cell Signaling Technology); antisynaptophysin (R\&D Systems); anti Ki67 (clone SP6, Spring Bioscience, Pleasanton, CA, USA). 2nd antibodies are: goat anti rabbit-Cy5; donkey anti rabbit- Cy3; donkey anti goat- Alexa Fluor 488 and donkey anti mouse- Cy3 (Jackson ImmunoResearch). In Situ Cell Death Detection Kit (Roche AG).

RAD001 (everolimus) (LC Laboratories, Woburn, MA, USA) was dissolved first in DMSO, following dilutions with PBS to yield a stock solution of $0.9 \mathrm{mg} / \mathrm{mL}$, which was stored at $-20^{\circ} \mathrm{C}$. Chloroquine was supplied by Sigma-Aldrich, Israel, and diluted in PBS to solution of $18 \mathrm{mg} / \mathrm{mL}$. CQ solution was prepared freshly every day, whereas RAD001 stock solution aliquots were thawed immediately before treating the animals. The controls were prepared as appropriate, using the same vehicle as for the drug (PBS).

\section{Subcutaneous xenograft mouse model}

Neoplasm xenografts were established 5 days following the subcutaneous injection of $4 \times 10^{6} \mathrm{BON} 1$ cells to the back of athymic nude mice (FOXN1NU NU/NU mice). Once neoplasm size reached $130 \mathrm{~mm}^{3}$, the mice were randomized into 4 groups and treated for the next 13 days with: (1) vehicle (PBS, $100 \mu \mathrm{L})$, (2) chloroquine (CQ) $(60 \mathrm{mg} / \mathrm{kg})$, (3) RAD001 (3 mg/kg), or (4) RAD001 and CQ (3 mg/kg and $60 \mathrm{mg} / \mathrm{kg}$, respectively). All treatments were administrated 
by i.p. (intra-peritoneal) injections. Neoplasm size was measured daily using a caliper, and neoplasm volume was calculated using the equation: length $\times\left(\right.$ width $\left.^{2}\right) / 2$. We have chosen the drug concentrations after reviewing the literature and conducting calibration experiments with the recommended concentrations in this model, and finally choosing those concentrations that suggested efficacy. The animals were treated daily (except for weekends). At the completion of the experiment, mice were killed and the neoplasms were taken for histopathologic analysis. An illustration of the subcutaneous xenograft model is presented in Fig. 1.

\section{Immunofluorescence staining}

All tissue and neoplasm samples were paraffinembedded, sectioned at $5 \mu \mathrm{m}$, deparaffinized, dehydrated and antigens retrieved. Sections of neoplasms went through blocking with CAS block (Life Technologies) for $10 \mathrm{~min}$, and stained with the following antibodies: anti-LC3-II; anti-phospho-S6; anti-SQSTM1/P62 (all purchased from Cell Signaling Technology) and antisynaptophysin (R\&D Systems); anti- Ki67 (clone SP6, Spring Bioscience). Slides were stained with the 1st antibody for $16 \mathrm{~h}$ in $4^{\circ} \mathrm{C}$, followed by wash and 2 -h incubation with one of the following 2nd antibodies: goat anti rabbit-Cy5; donkey anti rabbit- Cy3; donkey anti goat- 488; donkey anti mouse-Cy3 (all purchased from Jackson ImmunoResearch). Nuclear staining was done by DAPI (KPL, Gaithersburg, MD, USA) and covered with mounting solution (Immco Diagnostics
Inc, Buffalo, NY, USA). Slides were imaged with a $63 \times$ or $24 \times$ objective using a confocal microscope ZEISS LSM 710 (Carl Zeiss). Image quantification of phospho-S6 staining; P62 staining; cleaved caspase 3 staining and LC3-II puncta accumulation were conducted with ZEN light edition 2009 and ImageJ software.

\section{Assessment of neoplasm apoptosis}

Neoplasm samples were stained for TUNEL (TdT-mediated dUTP nick and labeling) using In Situ Cell Death Detection Kit (Roche AG) according to the manufacturer's instructions. Slides were imaged with a $63 \times$ objective using a confocal microscope ZEISS LSM 710 (Carl Zeiss). Image quantification of TUNEL positive cells was conducted with ZEN light edition 2009 and Image J software.

\section{Neoplasm necrosis assessment}

Neoplasm necrosis assessment was performed after H\&E staining and by calculating necrosis volume relative to total neoplasm volume for each neoplasm.

The study was approved by the Animal Care and Use Committee of the Hebrew University, Jerusalem, Israel (MD-14-13746-5).

\section{Statistical analysis}

Statistical analysis and significance of differences between groups were assessed by Student's $t$-test. The results are shown as mean \pm S.E.M. Significance was taken at $P<0.05$.

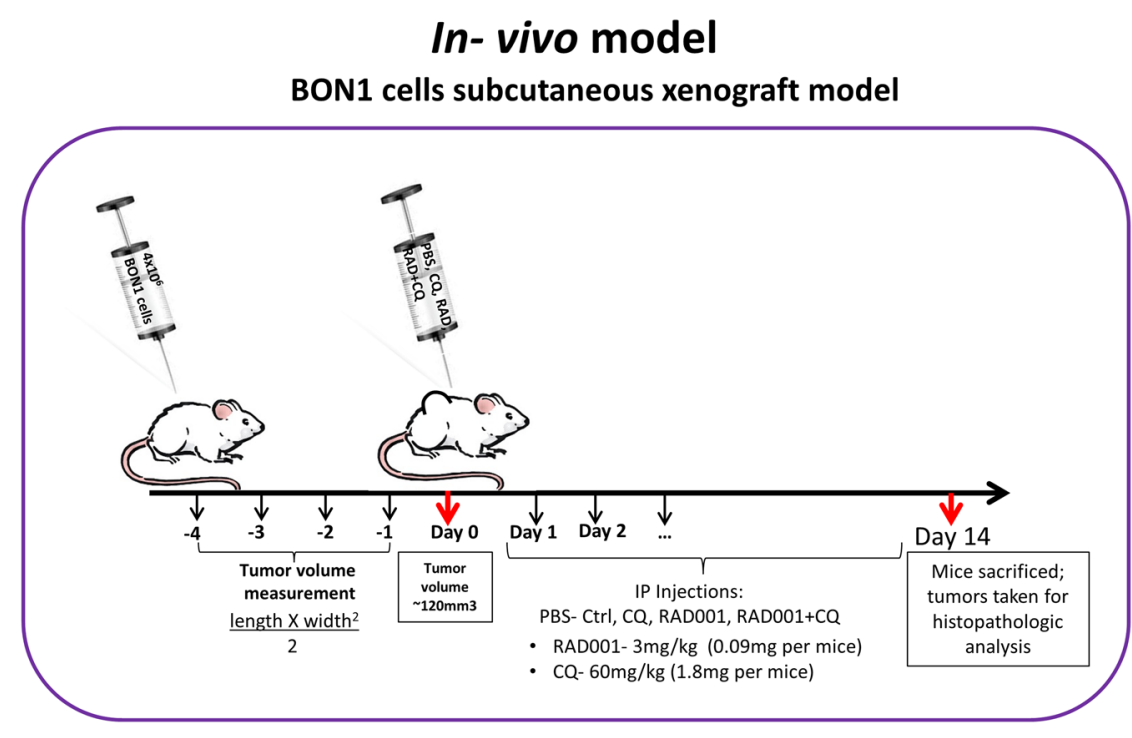

\section{In- vivo model}

Figure 1

An illustration of the subcutaneous xenograft mouse model. http://erc.endocrinology-journals.org https://doi.org/10.1530/ERC-18-0121
(C) 2018 Society for Endocrinology Published by Bioscientifica Ltd. Printed in Great Britain 


\section{Results}

The effect of chloroquine (CQ) alone or in combination with RAD001 on tumor size and weight

\section{CQ alone or in combination with RAD001}

significantly decreased tumor size in the BON1 subcutaneous NEN mouse model

As expected, mice treated with PBS developed large neoplasms, reaching an average size of $943 \mathrm{~mm}^{3}$ at the end of the study. Mice treated with CQ alone showed a decreased neoplasm size when compared to the control group (average size $588 \mathrm{~mm}^{3}$ ), indicating that CQ inhibited neoplasm growth. RAD001-treated group also developed smaller neoplasms than the control mice (average size $343 \mathrm{~mm}^{3}$ ). Noteworthy, the combination of CQ and RAD001 induced a marked reduction in neoplasm size, compared to drug given alone, reaching an average size of $223 \mathrm{~mm}^{3}$ at completion of the experiment (each neoplasm measurement was performed as related to its initial size) (Fig. 2A.). We monitored neoplasm volumes in the RAD001 and the RAD001+CQ groups for an extended period to test whether the effect on neoplasm size persists. The inhibitory effect of RAD001 persisted for up to $\sim 22$ days, when the tumor stopped to respond to the treatment and progressed rapidly; noteworthy, the effect of the RAD001+CQ combination persisted for longer (up to $\sim 30$ days) but did not completely prevent tumor growth over time (Fig. 2B.).

\section{CQ alone or in combination with RAD001 significantly reduced neoplasm weight in the BON1 subcutaneous neoplasm mouse model}

At the end of the experiment, mice were killed, and their neoplasms were excised and weighed. Mice from the control group developed larger neoplasms, reaching an average weight of $571 \mathrm{mg}$, while those from the CQ group and RAD001 group developed smaller neoplasms, reaching
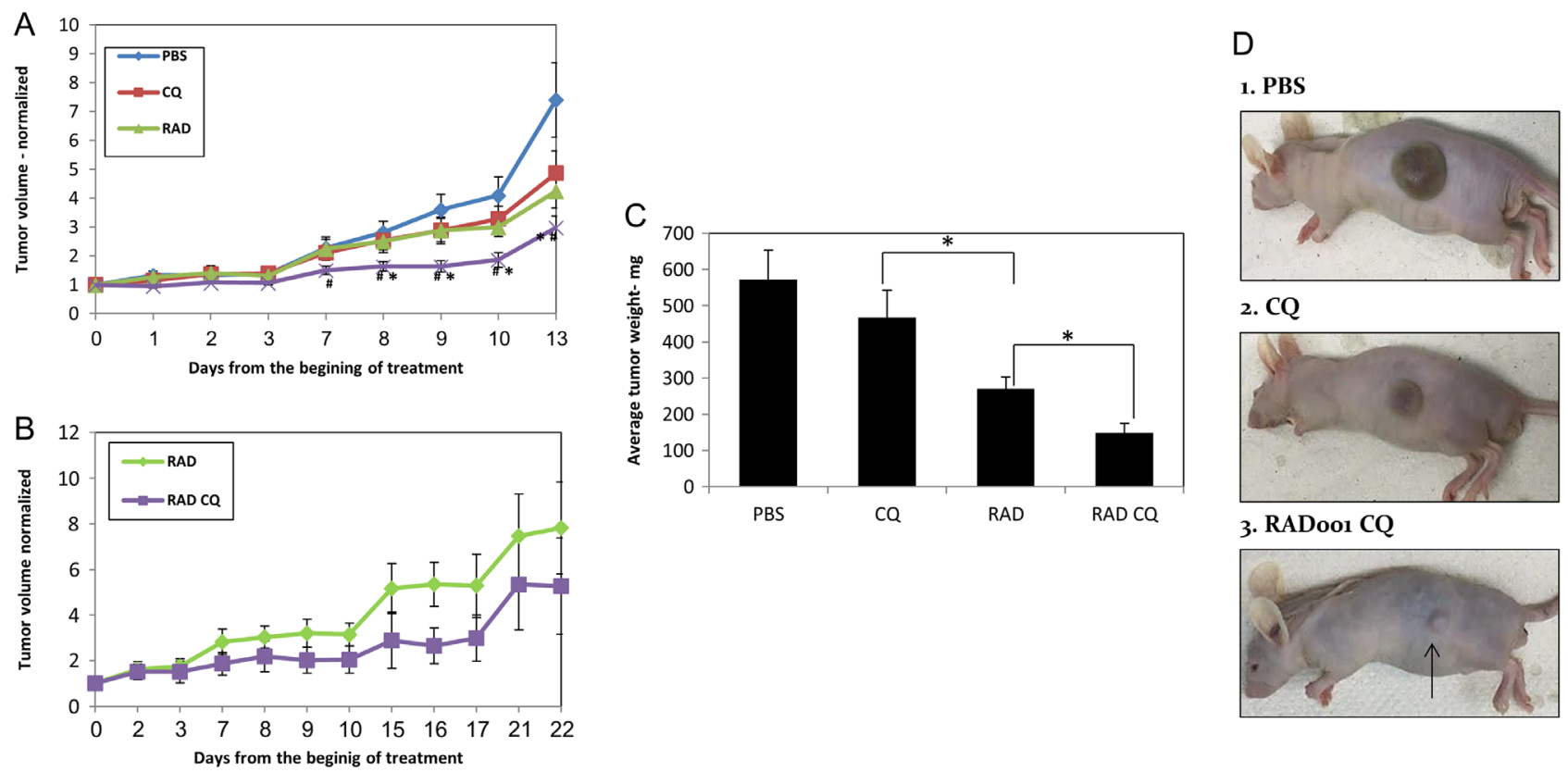

\section{Figure 2}

Administration of CQ with RAD001 significantly decreased tumor growth in BON1 subcutaneous neoplasm model. $4 \times 10^{6}$ BON1 cells were subcutaneously injected to athymic nude mice, which were then randomized and treated with the following: (1) vehicle (PBS, $100 \mu \mathrm{L}),(2) \mathrm{chloroquine}$ (CQ) $(60 \mathrm{mg} / \mathrm{kg})$, (3) RAD001 (3 mg/kg), (4) RAD001 and CQ (3 mg/kg and $60 \mathrm{mg} / \mathrm{kg}$ respectively), for the next 13 days. Tumor size was measured daily using caliper and tumor volume was calculated using the equation: length*(width2)/2. (A) Tumor volume measurements: PBS-treated group (blue line) developed large neoplasms; however, CQ (red line) and RAD001 (green line) showed an inhibitory effect on tumor growth with decrease in tumor size. In the RAD001 + CQ group (purple line), the decrease in the tumor size was more significant. PBS $(n=12)$, CQ $(n=9)$, RAD001 ( $n=11)$, RAD001 and CQ $(n=10)$. *RAD001 vs RAD CQ significance $P<0.05$, "CQ vs RAD CQ significance $P<0.05$. (B) Tumor volume - long-term follow-up: We followed tumor volume in the RAD001 and the RAD001 + CQ groups up to day 22. RAD001 (green line, $n=7$ ), RAD001 and CQ (purple line, $n=6$ ). The inhibitory effect of RAD001 + CQ treatment persisted for a longer period. (C) Average tumor weight summary: BON1 cells were subcutaneously injected to athymic nude mice as described. At the completion of the experiment, mice were killed, and tumors were excised and weighted. The figure summarizes the average of tumor weight in the treatments groups: PBS group: $571 \mathrm{mg}(n=11)$, CQ group: $467 \mathrm{mg}(n=9)$, RAD001 group: $271 \mathrm{mg}(n=10)$, RAD001 +CQ group: 148 mg $(n=9)$. Significant vs CQ $P<0.05$. (Fig. 3A). (D) Representative pictures of the tumors: (1) mouse treated with PBS, (2) mouse treated with CQ, (3) mouse treated with the combination of RAD001+CQ (arrows point to the tumor). 
an average weight of $467 \mathrm{mg}$ and $271 \mathrm{mg}$, respectively. Interestingly, mice treated with the combination of RAD001+CQ developed significantly smaller neoplasms compared to the other groups, reaching an average weight of $148 \mathrm{mg}$ (Fig. 2C). Representative pictures of neoplasms are presented in Fig. 2D.

\section{$C Q$ alone and in combination with RADO01 inhibits mTORC1 pathway signaling}

We next studied the effects of CQ, RAD001 and their combination on mTORC1 signaling by staining neoplasm sections for p-S6 kinase, a downstream target of mTORC1. In the control group, there was high expression of $\mathrm{p}-\mathrm{S} 6$ in neoplasm cells (Fig. 3A). Interestingly, the effect of RAD001 on p-S6 expression was mild (Fig. 3B), possibly reflecting a relative neoplasm resistance to rapalogs in this model. Moreover, CQ also decreased p-S6 staining (Fig. 3C). Noteworthy, treatment with CQ and RAD001 together synergistically inhibited mTORC1 evident by marked reduction of p-S6 in neoplasm cells (Fig. 3D and E). Collectively, these findings suggest that treatment with CQ facilitated the inhibition of mTORC1 by rapalogs.

\section{The combination of CQ with RAD001 arrests autophagy in mice neoplasms}

Upon stimulation of autophagy, cytosolic LC3 (LC3-I) is recruited to the autophagosome membrane and undergoes lipidation, resulting in the LC3-II form. LC3-II decorates the autophagosome membrane and can be used to quantify autophagosome number
(Mizushima \& Yoshimori 2007). LC3-II undergoes degradation in autolysosomes; therefore, treatment with lysosomal enzyme inhibitors, such as CQ, is expected to increase the number of LC3-II puncta (Ichimura et al. 2000, Kabeya et al. 2000, He et al. 2003, Kabeya et al. 2004, Tanida et al. 2004, Wu et al. 2006, Yang et al. 2011).

Tumor sections were stained for the autophagy marker LC3 and for the NEN marker synaptophysin. We are aware that the antibody used against LC3 in the immunofluorescence staining does not distinguish between LC3I and LC3II; however, we have previously shown that in BON1 cell line, both CQ and RAD001 + CQ induce mainly an increase in the amount of LC3II (Avniel-Polak et al. 2015). Neoplasms taken from the PBS group exhibited low levels of LC3 stain (Fig. 4A). As expected, neoplasms from the CQ-treated group exhibited high levels of LC3 (Fig. 4B), whereas neoplasms from the RAD001-treated group exhibited reduced levels of LC3. It is possible that RAD001 similarly stimulated autophagosome generation and turnover, hence preventing the accumulation of autophagosomes (and of LC3) (Fig. 4C.). However, neoplasms taken from the RAD001+CQ-treated group exhibited higher levels of LC3 (Fig. 4D) as the result of the combination of autophagy acceleration by RAD001 together with inhibition of lysosomal degradation by CQ. LC3 mean fluorescence intensity levels are shown in Fig. 4E.

However, as RAD001 only mildly inhibited mTORC1 whereas CQ potentiated RAD001 inhibition of mTORC1 (as demonstrated by the pS6 staining, Fig. 3), an alternative scenario could be that the neoplasm xenograft
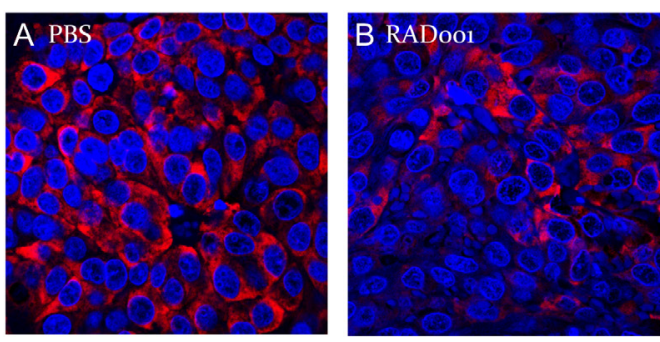

DAPI

P-S6
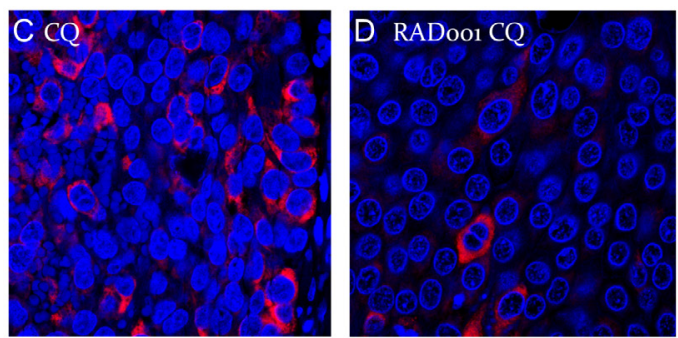

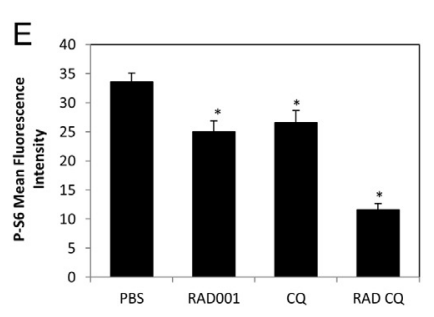

Figure 3

RAD001 + CQ in combination decreases p-S6 levels, indicating mTOR pathway inhibition. (A) Tumors treated with PBS exhibited high levels of $p$-S6. (B) Tumors treated with RAD001 exhibited a mild decrease in $\mathrm{p}-\mathrm{S6}$ levels, which was more significant in the tumors treated with CQ (C), suggesting a possible inhibitory effect of CQ on mTOR pathway. (D) Tumors treated with RAD001 +CQ exhibited a significant decrease in p-S6 levels compared to each one of the other groups. (E) p-S6 mean fluorescence intensity levels. 

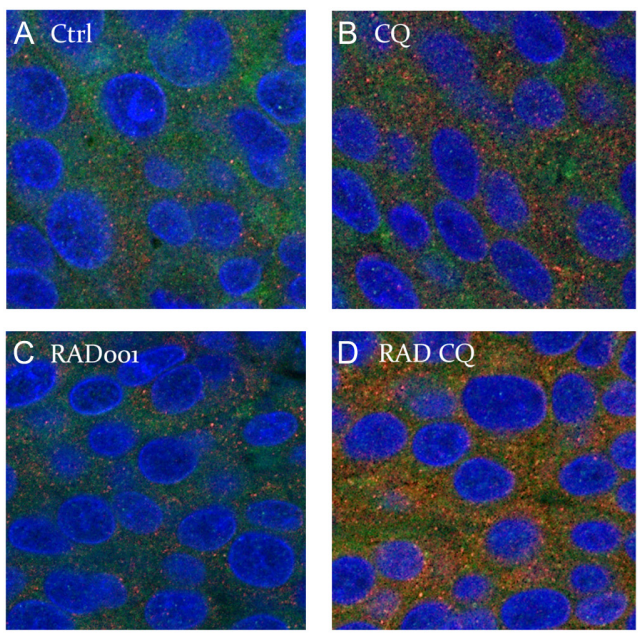

DAPI

$\mathrm{LC}_{3}$

Synaptophysin

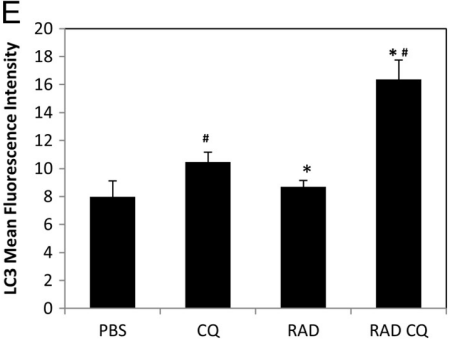

\section{Figure 4}

The combination of RAD001+CQ increases LC3 levels, indicating autophagy arrest. Mice neoplasm samples were stained for the autophagy marker LC3 (red) and for the NEN marker synaptophysin (green), followed by DAPI nuclear stain (blue). (A) Neoplasms from the PBS group exhibited low levels of LC3 stain. (B) CQ-treated neoplasms exhibited high levels of LC3. (C) RAD001-treated neoplasms exhibited reduced levels of LC3. (D) RAD001 + CQ-treated neoplasms exhibited significantly higher levels of LC3. (E) LC3 mean fluorescence intensity levels. was relatively resistant to the stimulatory effect of RAD001 on autophagy and that CQ-induced autophagy in RAD001-treated cells by inhibiting mTORC1. To differentiate between these two possibilities, we stained the neoplasm grafts for P62/SQSTM1 (Fig. 5). On the one hand, P62 is sequestered in autophagosomes being degraded in autolysosome; therefore, its expression may serve as a reporter of autophagic flux (Klionsky 2005); on the other hand, P62 is an integral part of the mTORC1 allowing its activation (Duran et al. 2011). P62/SQSTM1 expression was increased in both CQ- and RAD001+CQ-treated mice (Fig. 5B and D), indicating that CQ indeed inhibited lysosomal degradation in vivo. Intriguingly, RAD001 did not affect P62/SQSTM1 level in the neoplasm grafts compared to control mice (Fig. 5A and $\mathrm{C})$, suggesting that RAD001 alone had a limited effect on autophagy via mTORC1 in this neoplasm model.

\section{The combination of CQ with RADO01 increased neoplasm cells apoptosis}

In order to assess neoplasm cell proliferation, slides were stained for the Ki67 proliferation marker. As the Ki67 was high reaching 91-95\% in all neoplasms samples (irrespective of the treatment arm, data not shown), we assumed that the inhibition of neoplasm growth was not via a decrease in cells proliferation, but rather by inducing cell death. Therefore, our next step was to study the effect of CQ alone or in combination with RAD001 on neoplasm cell apoptosis and necrosis.

Apoptosis was assessed by TUNEL. Neoplasms treated with RAD001 but mainly with the combination of RAD001+CQ significantly increased the number of apoptotic cells (Fig. 6C and D) compared to CQ alone (Fig. 6A and B).

Neoplasm necrosis was assessed by H\&E staining; necrosis was present in all treatment groups, but mainly
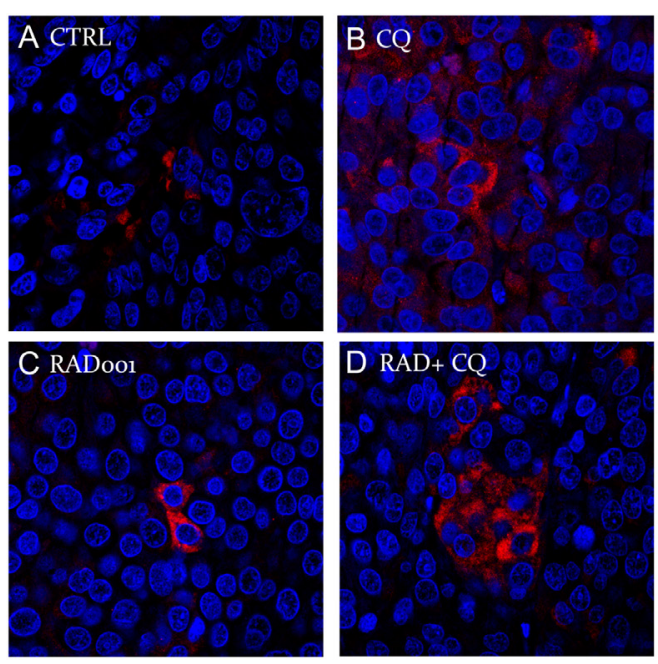

DAPI P62

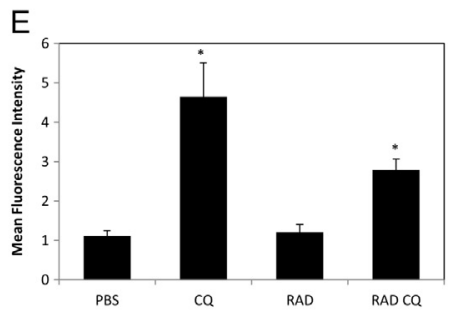

\section{Figure 5}

Examining autophagy flux by P62 staining revealed a limited effect of RAD001 on autophagy and a significant inhibition by $C Q$ of both autophagy and mTORC1. Mice neoplasm samples were stained for P62 (red) followed by DAPI (blue) nuclear stain. (A) Neoplasms treated with PBS presented a very weak P62 stain. (B) CQ-treated neoplasms presented significantly high levels of P62 staining. (C) Neoplasms treated with RAD001 did not affect P62 stain compared to control mice, suggesting that RAD001 alone has a limited effect on autophagy via mTORC1 in this model. (D) RAD001 + CQ-treated neoplasms presented high $\mathrm{P} 62$ staining, indicating that $\mathrm{CQ}$ indeed inhibited lysosomal degradation in vivo. (E) P62 mean fluorescence intensity levels. 

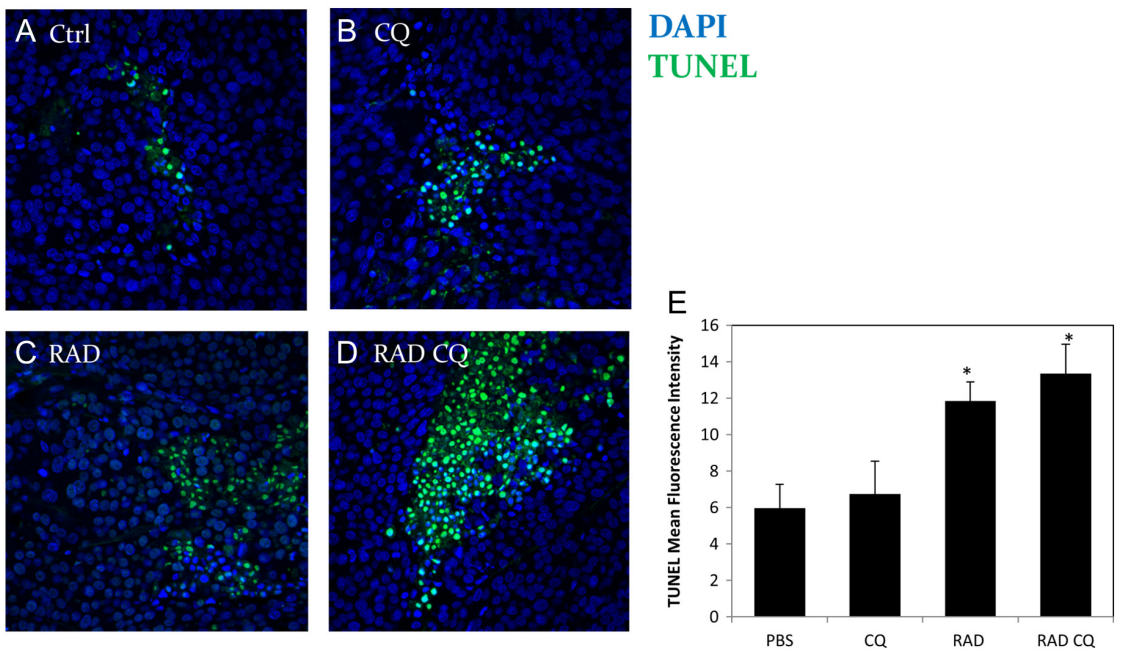

\section{Figure 6}

The combination of RAD001+CQ increases tumor cell apoptosis (TUNEL staining). Mice tumor samples were stained for TUNEL (green) followed by DAPI (blue) nuclear stain. (A) Tumors treated with PBS presented mild levels of apoptotic cells. (B) CQ treated tumors presented mild levels of apoptotic cells. (C) Tumors treated with RAD001 presented an increase in apoptotic cells.

(D) Tumors from RAD001 + CQ group presented significantly higher levels of apoptotic cells.

(E) TUNEL mean fluorescence intensity levels. increased in the CQ+RAD001-treated neoplasms (45\% with PBS, 50\% with CQ, 41\% with RAD001 and $62 \%$ with CQ+RAD001, respectively) (Fig. 7).

\section{Discussion}

In the current study, we demonstrated for the first time that, in the human pancreatic carcinoid (BON1) xenograft mouse model, CQ has by itself an anti-tumor effect, as seen by the decrease in tumor size and weight, and the addition of CQ to RAD001 showed even a stronger inhibitory effect. Based on these results, we looked further to understand the possible mechanisms by which $\mathrm{CQ}$, alone or combined with RAD001, exerts these effects. We and others have previously shown that CQ markedly inhibits autophagy and interferes with cell component degradation, inhibits cell proliferation and arrests cell cycle via downregulation of cyclin D1 (Avniel-Polak et al. 2015, Jia et al. 2017).
Here, we examined initially the p-S6 expression in the treated neoplasm samples, as a known downstream marker of the mTORC1 signaling pathway. Noteworthy, CQ administration significantly decreased p-S6 staining, suggesting that this drug inhibits mTOR pathway signaling and activation. Surprisingly, the inhibitory effect of RAD001 was mainly evident in the early stages of our study (up to day 13), when we had to interrupt the experiments as in the control group of mice treated with PBS the tumors reached the upper limit of the ethically permitted tumor' volume. However, when we continued to observe only the mice treated with RAD and RAD +CQ for an extended period of time (up to 22 days), we saw an increase in the levels of S6, possibly suggesting the development of a relative resistance to RAD001 in this specific model; however, the co-administration of CQ with RAD001 markedly inhibited mTORC1 activity, suggesting a synergistic inhibitory effect on mTOR signaling and a possible sensitizing effect of CQ on cell response to RAD001 treatment.
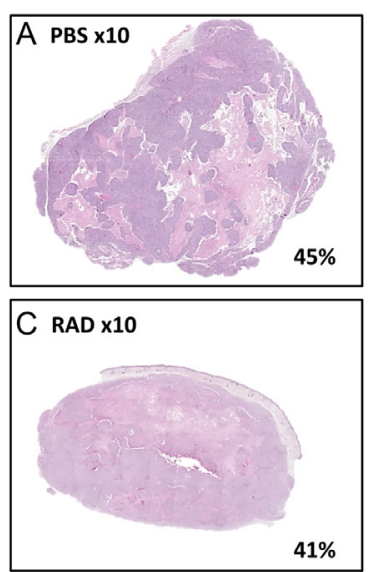
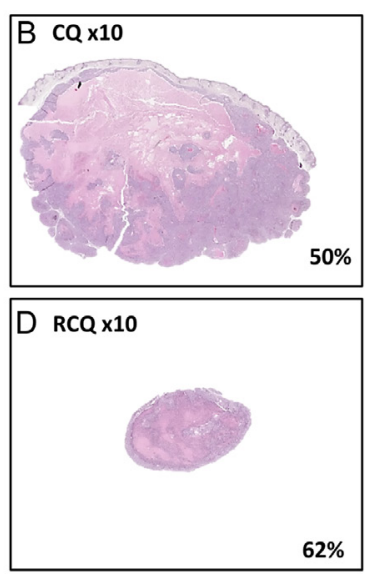

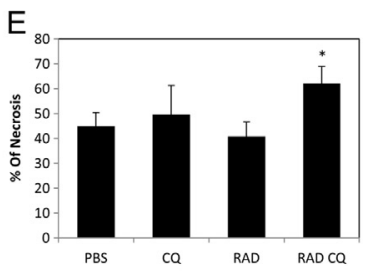

\section{Figure 7}

Tumor necrosis evaluation. Sections of tumor samples were stained for H\&E and evaluated for tumor cells necrosis which was present in all treatment groups (45\% with PBS) (A), 50\% with CQ (B), 41\% with RAD001 (C), and mainly increased in the RAD001 + CQ treated tumors (reaching 62\%) (D). 
We have previously demonstrated in an in vitro model that mTORi significantly increased the autophagic flux (Avniel-Polak et al. 2015). In the present study, we show that LC3-II accumulation (indicator of autophagy arrest) is increased in neoplasms treated with CQ. Moreover, as the effect of RAD001 was only mild on both autophagy arrest and mTORC1 inhibition, but increased when CQ was added, we believe that CQ most probably potentiated the effect of RAD001, suggesting an indirect effect of CQ on mTORC1. Since mTORC1 senses the amino acid concentration in the cytosol as well as in the lysosome lumen, we hypothesized that $C Q$, by inhibiting the lysosome catabolic activity, interferes with the aminoacid efflux to the cytosol, which may additionally inhibit mTORC1 through an indirect mechanism. This may explain the inhibitory effect of CQ on mTOR pathway as seen by the reduction in p-S6 activity following CQ treatment, as well as the synergistic inhibitory effect of CQ when added to RAD001; however, further studies are required to clarify these findings.

Since LC3-II correlates with the number of autophagosomes, elevation of LC3-II indicated autophagosomes accumulation and increased autophagy arrest. The increase in autophagosomes accumulation was reported to indicate a potential toxic effect on cells. For example, in a myocardial ischemia model, autophagosomes are formed but fail to be cleared, and their accumulation resulted in cell death (Geng et al. 2010, Marino et al. 2014). This effect has been previously reported also in insulinoma cells (Bachar-Wikstrom et al. 2013a) as well in other models (Geng et al. 2010, Choi et al. 2011). Moreover, the mutual interconnection between autophagy and cell death (apoptosis) is highly contextdependent. Autophagy may function as a guardian or executioner of apoptosis depending on the surrounding microenvironment, therapeutic intervention and the stage of a neoplasm (Li et al. 2017). In general, autophagy blocks the induction of apoptosis, and apoptosisassociated caspase activation inhibits the autophagic process (Marino et al. 2014). Noteworthy, in our study, we demonstrated both an induction in apoptosis and in necrosis in neoplasms treated with the combination of CQ and RAD001 (Figs 6 and 7).

In summary, we suggest that $\mathrm{CQ}$, alone and in addition to RAD001, arrests autophagy in NEN cells, induces autophagosomes accumulation with a toxic effect on the cells, and decreases mTOR signaling, hence inhibiting neoplasm growth. Moreover, we suggest a possible new mechanism explaining CQ inhibition of mTORC1 via the interruption of the amino-acid efflux and emphasizing the mTOR role as an amino acid sensor.

The main limitation of our study is the use of the highly proliferative BON1 xenograft neoplasm model, meaning that these results may not be directly applicable for the in vivo well-differentiated NEN models. BON1 is a well-known and highly proliferative cell line of human pancreatic carcinoid origin frequently used as a NEN in vitro model. Whereas our results demonstrated here using a BON1 xenograft mouse model might not be directly extrapolated to in vivo G1 or G2 NEN models, they might be even more suggestive for the possible efficacy of autophagy inhibition as a therapeutic target in NEN models with a lower proliferation rate. However, due to the rarity of well-differentiated NEN cell-lines (i.e. QGP-1 cell line), until such cell-lines will be widely available, researchers in the field will have to rely on studies such as the present one.

Our results are indeed encouraging and suggest that autophagy inhibitors, such as chloroquine, may represent a new therapeutic approach for patients with metastatic progressive NENs, both before and when progressing under mTORi treatment. Chloroquine seems to have potent anti-neoplasm effects in this NEN xenograft model and therefore it warrants further evaluation in clinical trials in patients with advanced NENs.

\section{Declaration of interest}

David J Gross receives honoraria for advisory board participation for Pfizer, Ipsen, Novartis, and Lexicon Pharmaceuticals, Inc., and study support from Novartis and Medison. Simona Grozinsky-Glasberg received speaker's honorarium, advisory board and educational research grants from Pfizer, Ipsen and Novartis and advisory board honorarium from Lexicon Pharmaceuticals.

\section{Funding}

This work was supported by a research grant provided by Novartis Oncology, Basel, Switzerland.

\section{Acknowledgements}

We would like to thank Dr Lola Weiss for her help in establishing the subcutaneous NEN xenograft model.

\section{References}

Altomare DA \& Testa JR 2005 Perturbations of the AKT signaling pathway in human cancer. Oncogene 24 7455-7464. (https://doi. org/10.1038/sj.onc.1209085)
2018 Society for Endocrinology Published by Bioscientifica Ltd. Printed in Great Britain 
Amaravadi RK, Yu D, Lum JJ, Bui T, Christophorou MA, Evan GI, Thomas-Tikhonenko A \& Thompson CB 2007 Autophagy inhibition enhances therapy-induced apoptosis in a Myc-induced model of lymphoma. Journal of Clinical Investigation 117 326-336. (https://doi. org/10.1172/JCI28833)

Amaravadi R, Kimmelman AC \& White E 2016 Recent insights into the function of autophagy in cancer. Genes and Development 30 1913-1930. (https://doi.org/10.1101/gad.287524.116)

Avniel-Polak S, Leibowitz G, Riahi Y, Glaser B, Gross DJ \& GrozinskyGlasberg S 2015 Abrogation of autophagy by chloroquine alone or in combination with mTOR inhibitors induces apoptosis in neuroendocrine tumor cells. Neuroendocrinology 103 724-737. (https://doi.org/10.1159/000442589)

Bachar-Wikstrom E, Wikstrom JD, Ariav Y, Tirosh B, Kaiser N, Cerasi E \& Leibowitz G 2013a Stimulation of autophagy improves endoplasmic reticulum stress-induced diabetes. Diabetes 62 1227-1237. (https:// doi.org/10.2337/db12-1474)

Bachar-Wikstrom E, Wikstrom JD, Kaiser N, Cerasi E \& Leibowitz G $2013 b$ Improvement of ER stress-induced diabetes by stimulating autophagy. Autophagy 9 626-628. (https://doi.org/10.4161/ auto.23642)

Cecconi F \& Levine B 2008 The role of autophagy in mammalian development: cell makeover rather than cell death. Developmental Cell 15 344-357. (https://doi.org/10.1016/j.devcel.2008.08.012)

Choi J, Jo M, Lee E \& Choi D 2011 Induction of apoptotic cell death via accumulation of autophagosomes in rat granulosa cells. Fertility and Sterility 95 1482-1486. (https://doi.org/10.1016/j. fertnstert.2010.06.006)

Czarny P, Pawlowska E, Bialkowska-Warzecha J, Kaarniranta K \& Blasiak J 2015 Autophagy in DNA damage response. International Journal of Molecular Sciences 16 2641-2662. (https://doi.org/10.3390/ ijms16022641)

Dasari A, Shen C, Halperin D, Zhao B, Zhou S, Xu Y, Shih T \& Yao JC 2017 Trends in the incidence, prevalence, and survival outcomes in patients with neuroendocrine tumors in the United States. JAMA Oncology 3 1335-1342. (https://doi.org/10.1001/ jamaoncol.2017.0589)

Degtyarev M, De Maziere A, Orr C, Lin J, Lee BB, Tien JY, Prior WW, van Dijk S, Wu H, Gray DC, et al. 2008 Akt inhibition promotes autophagy and sensitizes PTEN-null tumors to lysosomotropic agents. Journal of Cell Biology 183 101-116. (https:/doi.org/10.1083/ jcb.200801099)

Duran A, Amanchy R, Linares JF, Joshi J, Abu-Baker S, Porollo A, Hansen M, Moscat J \& Diaz-Meco MT 2011 p62 is a key regulator of nutrient sensing in the mTORC1 pathway. Molecular Cell 44 134-146. (https://doi.org/10.1016/j.molcel.2011.06.038)

Fan QW, Cheng C, Hackett C, Feldman M, Houseman BT, Nicolaides T, Haas-Kogan D, James CD, Oakes SA, Debnath J, et al. 2010 Akt and autophagy cooperate to promote survival of drug-resistant glioma. Science Signaling 3 ra81.

Fang Y, Tan J \& Zhang Q 2015 Signaling pathways and mechanisms of hypoxia-induced autophagy in the animal cells. Cell Biology International 39 891-898. (https://doi.org/10.1002/cbin.10463)

Ganley IG, Lam du H, Wang J, Ding X, Chen S \& Jiang X 2009 ULK1. ATG13.FIP200 complex mediates mTOR signaling and is essential for autophagy. Journal of Biological Chemistry 284 12297-12305. (https:// doi.org/10.1074/jbc.M900573200)

Geng Y, Kohli L, Klocke BJ \& Roth KA 2010 Chloroquine-induced autophagic vacuole accumulation and cell death in glioma cells is p53 independent. Neuro-Oncology 12 473-481.

Grozinsky-Glasberg S \& Pavel M 2012 Inhibition of mTOR in carcinoid tumors. Targeted Oncology 7 189-195. (https://doi.org/10.1007/ s11523-012-0225-x)

Grozinsky-Glasberg S, Grossman AB \& Korbonits M 2008 The role of somatostatin analogues in the treatment of neuroendocrine tumours.
Molecular and Cellular Endocrinology 286 238-250. (https://doi. org/10.1016/j.mce.2007.10.006)

Grozinsky-Glasberg S, Rubinfeld H, Nordenberg Y, Gorshtein A, Praiss M, Kendler E, Feinmesser R, Grossman AB \& Shimon I 2010 The rapamycin-derivative RAD001 (everolimus) inhibits cell viability and interacts with the Akt-mTOR-p70S6K pathway in human medullary thyroid carcinoma cells. Molecular and Cellular Endocrinology $\mathbf{3 1 5}$ 87-94. (https://doi.org/10.1016/j.mce.2009.09.027)

He H, Dang Y, Dai F, Guo Z, Wu J, She X, Pei Y, Chen Y, Ling W, Wu C, et al. 2003 Post-translational modifications of three members of the human MAP1LC3 family and detection of a novel type of modification for MAP1LC3B. Journal of Biological Chemistry 278 29278-29287. (https://doi.org/10.1074/jbc.M303800200)

Hong SK, Kim JH, Starenki D \& Park JI 2013 Autophagy sensitivity of neuroendocrine lung tumor cells. International Journal of Oncology $\mathbf{4 3}$ 2031-2038. (https://doi.org/10.3892/ijo.2013.2136)

Hosokawa N, Hara T, Kaizuka T, Kishi C, Takamura A, Miura Y, Iemura S, Natsume T, Takehana K, Yamada N, et al. 2009 Nutrient-dependent mTORC1 association with the ULK1-Atg13-FIP200 complex required for autophagy. Molecular Biology of the Cell 20 1981-1991. (https:// doi.org/10.1091/mbc.E08-12-1248)

Ichimura Y, Kirisako T, Takao T, Satomi Y, Shimonishi Y, Ishihara N, Mizushima N, Tanida I, Kominami E, Ohsumi M, et al. 2000 A ubiquitin-like system mediates protein lipidation. Nature $\mathbf{4 0 8}$ 488-492. (https://doi.org/10.1038/35044114)

Jia L, Wang J, Wu T, Wu J, Ling J \& Cheng B 2017 In vitro and in vivo antitumor effects of chloroquine on oral squamous cell carcinoma. Molecular Medicine Reports 16 5779-5786. (https://doi.org/10.3892/ mmr.2017.7342)

Jung CH, Jun CB, Ro SH, Kim YM, Otto NM, Cao J, Kundu M \& Kim DH 2009 ULK-Atg13-FIP200 complexes mediate mTOR signaling to the autophagy machinery. Molecular Biology of the Cell 20 1992-2003. (https://doi.org/10.1091/mbc.E08-12-1249)

Kabeya Y, Mizushima N, Ueno T, Yamamoto A, Kirisako T, Noda T, Kominami E, Ohsumi Y \& Yoshimori T 2000 LC3, a mammalian homologue of yeast Apg8p, is localized in autophagosome membranes after processing. EMBO Journal 19 5720-5728. (https:// doi.org/10.1093/emboj/19.21.5720)

Kabeya Y, Mizushima N, Yamamoto A, Oshitani-Okamoto S, Ohsumi Y \& Yoshimori T 2004 LC3, GABARAP and GATE16 localize to autophagosomal membrane depending on form-II formation. Journal of Cell Science 117 2805-2812. (https://doi.org/10.1242/jcs.01131)

Klionsky DJ 2005 The correct way to monitor autophagy in higher eukaryotes. Autophagy 1 65. (https://doi.org/10.4161/ auto.1.2.1899)

Li CJ, Liao WT, Wu MY \& Chu PY 2017 New insights into the role of autophagy in tumor immune microenvironment. International Journal of Molecular Sciences 18 E1566. (https://doi.org/10.3390/ ijms18071566)

Liu G, Pei F, Yang F, Li L, Amin AD, Liu S, Buchan JR \& Cho WC 2017 Role of autophagy and apoptosis in non-small-cell lung cancer. International Journal of Molecular Sciences 18 367. (https://doi. org/10.3390/ijms18020367)

Marino G, Niso-Santano M, Baehrecke EH \& Kroemer G 2014 Selfconsumption: the interplay of autophagy and apoptosis. Nature Reviews Molecular Cell Biology 15 81-94. (https://doi.org/10.1038/ nrm3735)

Mazure NM \& Pouyssegur J 2010 Hypoxia-induced autophagy: cell death or cell survival? Current Opinion in Cell Biology 22 177-180. (https:// doi.org/10.1016/j.ceb.2009.11.015)

Mizushima N \& Yoshimori T 2007 How to interpret LC3 immunoblotting. Autophagy 3 542-545. (https://doi.org/10.4161/ auto.4600)

O'Reilly KE, Rojo F, She QB, Solit D, Mills GB, Smith D, Lane H, Hofmann F, Hicklin DJ, Ludwig DL, et al. 2006 mTOR inhibition
(2) 2018 Society for Endocrinology Published by Bioscientifica Ltd. Printed in Great Britain 
induces upstream receptor tyrosine kinase signaling and activates Akt. Cancer Research 66 1500-1508.

Oberg K 2005 Neuroendocrine tumors of the gastrointestinal tract: recent advances in molecular genetics, diagnosis, and treatment. Current Opinion in Oncology 17 386-391. (https://doi.org/10.1097/01. cco.0000167739.56948.a9)

Oberg K 2011 Neuroendocrine tumors: recent progress in diagnosis and treatment. Endocrine-Related Cancer 18 (Supplement 1) E3-E6. (https://doi.org/10.1530/ERC-10-0288)

Peterson TR, Laplante M, Thoreen CC, Sancak Y, Kang SA, Kuehl WM, Gray NS \& Sabatini DM 2009 DEPTOR is an mTOR inhibitor frequently overexpressed in multiple myeloma cells and required for their survival. Cell 137 873-886. (https://doi.org/10.1016/j.cell.2009.03.046)

Riahi Y, Wikstrom JD, Bachar-Wikstrom E, Polin N, Zucker H, Lee MS, Quan W, Haataja L, Liu M, Arvan P, et al. 2016 Autophagy is a major regulator of beta cell insulin homeostasis. Diabetologia $\mathbf{5 9}$ 1480-1491. (https://doi.org/10.1007/s00125-016-3868-9)

Rubinsztein DC, Codogno P \& Levine B 2012 Autophagy modulation as a potential therapeutic target for diverse diseases. Nature Reviews Drug Discovery 11 709-730. (https://doi.org/10.1038/nrd3802)

Seitz C, Hugle M, Cristofanon S, Tchoghandjian A \& Fulda S 2013 The dual PI3K/mTOR inhibitor NVP-BEZ235 and chloroquine synergize to trigger apoptosis via mitochondrial-lysosomal cross-talk. International Journal of Cancer 132 2682-2693. (https://doi. org/10.1002/ijc.27935)
Tanida I, Ueno T \& Kominami E 2004 Human light chain 3/MAP1LC3B is cleaved at its carboxyl-terminal Met121 to expose Gly120 for lipidation and targeting to autophagosomal membranes. Journal of Biological Chemistry 279 47704-47710. (https://doi.org/10.1074/jbc. M407016200)

Weckman A, Rotondo F, Di Ieva A, Syro LV, Butz H, Cusimano MD \& Kovacs K 2015 Autophagy in endocrine tumors. EndocrineRelated Cancer 22 R205-R218. (https://doi.org/10.1530/ERC-150042)

Wu J, Dang Y, Su W, Liu C, Ma H, Shan Y, Pei Y, Wan B, Guo J \& Yu L 2006 Molecular cloning and characterization of rat LC3A and LC3B - two novel markers of autophagosome. Biochemical and Biophysical Research Communications 339 437-442. (https://doi.org/10.1016/j. bbrc.2005.10.211)

Xu CX, Zhao L, Yue P, Fang G, Tao H, Owonikoko TK, Ramalingam SS, Khuri FR \& Sun SY 2011 Augmentation of NVP-BEZ235's anticancer activity against human lung cancer cells by blockage of autophagy. Cancer Biology and Therapy 12 549-555. (https://doi.org/10.4161/ cbt.12.6.16397)

Yang Z \& Klionsky DJ 2010 Mammalian autophagy: core molecular machinery and signaling regulation. Current Opinion in Cell Biology 22 124-131. (https://doi.org/10.1016/j.ceb.2009.11.014)

Yang ZJ, Chee CE, Huang S \& Sinicrope FA 2011 The role of autophagy in cancer: therapeutic implications. Molecular Cancer Therapeutics 10 1533-1541. (https://doi.org/10.1158/1535-7163.MCT-11-0047)

Received in final form 7 April 2018

Accepted 10 April 2018

Accepted Preprint published online 10 April 2018
(C) 2018 Society for Endocrinology Published by Bioscientifica Ltd. Printed in Great Britain 